\title{
ANÁLISE DO DESEMPENHO DE UM MODELO TURBULENTO VISCOELÁSTICO BASEADO NA EQUAÇÃO CONSTITUIVA GNF CONTRA O MODELO FENE-P
}

\author{
P. R. RESENDE ${ }^{1}$. \\ 1. Grupo de Automação e Sistemas Integráveis, UNESP - Univ. Estadual Paulista \\ 18087-180, Sorocaba-SP, Brazil \\ E-mails: resende@sorocaba.unesp.br
}

\begin{abstract}
Resumo- Um modelo turbulento de tensões de Reynolds para fluidos viscoelásticos, descrito pelo o modelo de fluidos newtoniano generalizado (GNF) é analisado em um escoamento turbulento entre placas com fluidos viscoelásticos. O modelo GNF foi modificado para capturar o efeito viscoelástico em escoamentos turbulentos através da introdução de uma viscosidade não linear que depende do reoespessamento da razão de Trouton. O seu desempenho é analisado comparando as previsões do modelo com as previsões da simulação numérica direta (DNS) de fluidos teóricos descritos pelo o modelo não linear de extensão finita usando a aproximação de Peterlin, designado pelo modelo FENE-P.
\end{abstract}

Palavras-chave— Modelo turbulento, redução de arrasto, modelo FENE-P.

\section{Introdução}

A redução do arrasto de fluidos viscoelásticos foi referida pela primeira vez por Toms em 1949. No seu trabalho usaram-se soluções diluídas de polímero tendo-se medido a relação entre a perda de carga e o caudal em escoamento turbulento em condutas. Desde essa altura o fenómeno tem atraído a atenção de muitos pesquisadores empenhados em descobrir os mecanismos que levam ao fenómeno, devido ao seu potencial em aplicações práticas de engenharia, tais como em sistemas de aquecimento e arrefecimento comunitário ou de transporte de fluidos a longas distâncias. Pinho (2003)

A necessidade de possuir ferramentas de cálculo numérico para aplicações de engenharia comuns, levou ao aparecimento dos primeiros modelos turbulentos para fluidos não newtonianos nos anos 70, como por exemplo, Mizushina et al. (1973), Durst e Rastogi (1977), Poreh e Hassid (1977) e Hassid e Poreh (1978). Estes modelos eram muito limitados visto que não se baseavam numa equação constitutiva e dependiam de parâmetros que mudavam consoante o tipo de geometria e fluido. Os primeiros modelos com uma contribuição elástica de uma forma generalizada foram os modelos turbulentos viscoelásticos de Cruz e Pinho (2003); Cruz et al. (2004); Pinho (2003); Resende et al. (2006) e Resende et al. (2013a), estes modelos eram baseados na equação constitutiva de um fluido newtoniano generalizado, que foi modificada através da introdução de uma dependência do segundo e terceiro invariante da taxa de deformação instantânea na viscosidade, sendo que o terceiro invariante é indexado à razão de Trouton, isto é, ao efeito da viscosidade extensional. Note-se que apesar de não existir uma teoria concreta para explicar o fenômeno da redução do arrasto, as pesquisas demonstram que existe uma ligação direta do aumento da redução do arrasto ao efeito reoespessante da viscosidade extensional.

Outra ferramenta de cálculo numérico é a simulação numérica directa (DNS), que tem a vantagem de ser mais precisa visto não necessitar de modelação para resolução das equações. Contudo, o seu elevado esforço computacional, não recomenda a sua aplicação directa a problemas de engenharia. Apesar disso, o DNS está a contribuir para o desenvolvimento dos modelos turbulentos através da apresentação detalhada da dinâmica do escoamento turbulento de fluidos viscoelásticos, Sureskumar et al. Sureshkumar et al. (1997), Dimitropoulos et al. Dimitropoulos et al. (2001), entre outros. Usando os dados de DNS para fluidos FENE-P, foram desenvolvidos vários modelos turbulentos, como por exemplo, o fecho da viscosidade turbulenta de Li et al. (2006a), o modelo $k$ - de Pinho et al. (2008) e Resende et al. (2011), o modelo $k$ - $\omega$ de Resende et al. (2013b), e o $k-v^{2}-f$ de Dubief et al. (2004) e Iaccarino et al. (2010).

Apesar da evolução dos modelos turbulentos para uma equação constitutiva realmente elástica existem ainda algumas limitações na previsão real de um escoamento turbulento com um fluido viscoelástico. Ptasinski et al. (2003) demonstrou que as previsões do DNS usando o modelo FENE-P não correspondiam aos dados obtidos experimentalmente, isto é, para os mesmos parâmetros de reologia e medições do escoamento existia uma diferença significativa em termos da redução de arrasto. Essa diferença será replicada nos modelos turbulentos baseados no FENE-P, e por essa razão os modelos mais simples, como por exemplo o GNF, 
desenvolvidos com recurso a dados experimentais, permitem obter resultados mais fiáveis.

Para entender melhor as limitações do modelo GNF, no presente trabalho as previsões do modelo turbulento de tensões de Reynolds, Resende et al. (2013a), são analisadas contra os dados do DNS descritos pela equação constitutiva FENE-P, após a comparação dos parâmetros reológicos definidos por ambos os modelos.

\section{Equações governativas}

Nas variáveis seguintes, as letras maiúsculas e com traço são definidas como quantidades médias de Reynolds e as minúsculas para flutuações. A notação Einstein será usada no artigo.

As equações governativas das quantidades médias de Reynols para um fluido incompressível são a equação da continuidade

$$
\frac{\partial U_{i}}{\partial x_{i}}=0
$$

e a equação de movimento

$$
\begin{aligned}
& \rho \frac{\partial U_{i}}{\partial t}+\rho U_{k} \frac{\partial U_{i}}{\partial x_{k}}=-\frac{\partial \bar{p}}{\partial x_{i}}+ \\
& \frac{\partial}{\partial x_{k}}\left(2 \bar{\mu} S_{i k}-\rho \overline{u_{i} u_{k}}+2 \overline{\mu^{\prime} s_{i k}}\right)
\end{aligned}
$$

onde $p$ é a pressão, $\bar{\mu}$ é a viscosidade média molecular, $u_{i}$ é a componente $i$ - do vector de velocidade e e $S_{i j}$ is é o tensor da taxa de deformação definido como $S_{i j} \equiv\left(\partial u_{i} / \partial x_{j}+\partial u_{j} / \partial x_{i}\right) / 2$.

A equação constitutiva usada na equação de movimento foi uma modificação do Fluido Newtoniano Generalizado desenvolvida anteriormente por Pinho (2003) usando as equações seguintes, Eq. (3) e (4).

$$
\begin{gathered}
\sigma_{i j}=\mu(\dot{\gamma}, \dot{\varepsilon}) S_{i j} \\
\mu(\dot{\gamma}, \dot{\varepsilon})=\underbrace{K_{v}\left[\dot{\gamma}^{2}\right]^{(n-1) / 2}}_{\eta_{v}} \underbrace{K_{e}\left[\dot{\varepsilon}^{2}\right]^{(p-1) / 2}}_{\eta_{e}^{*}}
\end{gathered}
$$

A presente equação constitutiva foi modificada para incluir efeitos não newtonianos em escoamentos turbulentos de fluidos viscoelásticos através da introdução do efeito reoespessante da viscosidade extensional. Os parâmetros $K_{v}$ e $n$ da aproximação da lei de potência aos dados da viscosidade de corte usando o invariante do tensor da taxa de deformação $\dot{\gamma}=\sqrt{2 S_{i j} S_{i j}}$. A contribuição reoespessante $\left(\eta_{e}^{*}\right)$ é definido com base da razão adimensional de Trouton $\eta_{e}(\dot{\varepsilon}) /\left(3 \eta_{v}(\dot{\gamma})\right)$ e os parâmetros $K_{e}$ e $p$ representam os parâmetros de aproximação aos dados da razão de Trouton, que depende da medição da reologia dos dos fluidos viscoelásticos. O outro invariante do tensor de deformação é quantificado pela relação $\dot{\varepsilon}=\dot{\gamma} / \sqrt{3}$, explicado por Barnes et al. (1989). Mais detalhes pdem ser encontrados em Pinho (2003) e Cruz e Pinho (2003).
A viscosidade média molecular, dada pela Eq. (5), é afetada pela turbulência, e combina a contribuição da viscosidade de corte pura, $\eta_{v}$, definida na Eq. (4), com a contribuição da viscosidade média de Reynolds para elevados números de Reynolds $\left(\bar{\mu}_{h}\right)$ da Eq. (6).

$$
\begin{gathered}
\bar{\mu}=f_{v} \bar{\mu}_{h}+\left(1-f_{v}\right) \eta_{v} \\
\bar{\mu}_{h}=\left(C_{\mu} \rho\right)^{3 m(m-1) A_{2} /\left(8+3 m(m-1) A_{2}\right)} \times 2^{4 m(m-1) A_{2} /\left(8+3 m(m-1) A_{2}\right)} \\
\times k^{6 m(m-1) A_{2} /\left(8+3 m(m-1) A_{2}\right)} \times \varepsilon^{\left(\left[8-3(m-1) A_{2}\right] m\right) /\left(8+3 m(m-1) A_{2}\right)} \\
\times B^{8 /\left(8+3 m(m-1) A_{2}\right)}
\end{gathered}
$$

onde $\varepsilon$ é a taxa de dissipação da energia cinética de turbulência $(k) \mathrm{e}$

$$
m=\frac{n+p-2}{n+p} ; B=\left[\frac{K_{v} K_{e}}{A_{\varepsilon}^{p-1}}\right]^{1-m} 2^{[(n-1)-m(n+1)] / 2} \rho^{m}
$$

A função de amortecimento, $f_{v}$, é definida na Eq. (8), e é igual à função de amortecimento $f$ da viscosidade turbulenta do modelo de turbulência $k-\varepsilon$ de Cruz e Pinho (2003) e Cruz et al. (2004). Os parâmetros restantes tomam os seguintes valores $A_{\varepsilon}=10$, $A_{2}=0.45$ e $C_{\mu}=0.084$.

$$
\begin{aligned}
& f_{v}=\left\{1-\left[1+\left|\frac{1-n}{1+n}\right| y^{+}\right]^{-|1+n / 1-n| / A^{+}}\right\} \\
& \times\left\{1-\left[1+\left|\frac{p-1}{3-p}\right| y^{+} C^{\frac{1-p}{2-p}}\right]^{-|3-p / p-1| / A^{+}}\right.
\end{aligned}
$$

A equação de movimento, Eq. (2), contém o divergente da tensão molecular, a tensão de Reynols e uma nova tensão não newtoniana designada por tensão pseudo-elástica, definida por

$$
2 \overline{\mu^{\prime} s_{i j}}=\tilde{C} \frac{K_{v} K_{e}}{A_{\varepsilon}{ }^{p-1}}\left[\left|\frac{\rho \overline{u_{i} u_{j}}}{4 \bar{\mu}} S_{i j}\right|\right]^{\frac{(p+n-2)}{2}} \times \frac{1}{L_{c}} \times \frac{\overline{u_{i} u_{j}}}{\sqrt{\mid \overline{u_{i} u_{j} \mid}}}(9)
$$

com

$$
\tilde{C}=\left(1+C_{0}\right)^{p+n-2}-1 .
$$

A tensão pseudo-elástica desaparece no limite newtoniano $(n=1$ e $p=1)$ como foi estabelecido, em termos do efeito elástico é contabilizado através do parâmetro $\tilde{C}$, que depende de um outro parâmetro $C_{0}=$ $-0.95$

O tensor das tensões de Reynolds $\left(\rho \overline{u_{i} u_{k}}\right)$ é determinado pela seguinte equação de transporte modelada para fluidos viscoelásticos. 


$$
\begin{aligned}
& \rho \frac{D \overline{u_{i} u_{j}}}{D t}+\rho \overline{u_{j} u_{k}} \frac{\partial U_{i}}{\partial x_{k}}+\rho \overline{u_{i} u_{k}} \frac{\partial U_{j}}{\partial x_{k}} \\
& =\rho \frac{\partial}{\partial x_{k}}\left\{C _ { s } \frac { k } { \varepsilon } \left[\overline{u_{i} u_{l}} \frac{\partial \overline{u_{j} u_{k}}}{\partial x_{l}}+\overline{u_{j} u_{l}} \frac{\partial \overline{u_{k} u_{i}}}{\partial x_{l}}\right.\right. \\
& \left.\left.+\overline{u_{k} u_{l}} \frac{\partial \overline{u_{i} u_{j}}}{\partial x_{l}}\right]\right\}+\underbrace{\phi_{i j, 1}+\phi_{i j, w} f_{w, 1}}_{\phi_{i j}^{*}}+\rho \varepsilon_{i j}+\bar{\mu} \frac{\partial^{2} \overline{u_{i} u_{j}}}{\partial x_{k} \partial x_{k}} \\
& +C_{V 1} \times \frac{\partial \bar{\mu}}{\partial x_{k}} \frac{\partial \overline{u_{i} u_{j}}}{\partial x_{k}}+C_{V 2} \times \frac{\partial \bar{\mu}}{\partial x_{k}}\left(\frac{\partial \overline{u_{k} u_{j}}}{\partial x_{i}}+\frac{\partial \overline{u_{k} u_{i}}}{\partial x_{j}}\right)
\end{aligned}
$$

com o fecho de $\varepsilon_{i j}$ dado por

$$
\begin{aligned}
& \varepsilon_{i j}=\frac{2}{3} \varepsilon\left(1-f_{w, 1}\right) \delta_{i j}+f_{w, 1}(\varepsilon / k) \\
& \times \frac{\left[\overline{u_{i} u_{j}}+\overline{u_{i} u_{k}} n_{k} n_{j}+\overline{u_{j} u_{k}} n_{k} n_{i}+n_{i} n_{j} \overline{u_{k} u_{l}} n_{k} n_{l}\right]}{1+3 \overline{u_{k} u_{l}} n_{l} n_{k} / 2 k}
\end{aligned}
$$

em que a ligação à reologia do fluido é feita através da função de amortecimento $f_{w, 1}$. O modelo para tensor da taxa de dissipação é definida pela Eq. (13).

$$
\begin{aligned}
& \frac{D \varepsilon}{D t}=\frac{\partial}{\partial x_{k}}\left(\bar{v} \frac{\partial \varepsilon}{\partial x_{k}}\right)+\frac{\partial}{\partial x_{k}}\left(C_{s} \frac{k}{\varepsilon} \overline{u_{k} u_{i}} \frac{\partial \varepsilon}{\partial x_{i}}\right) \\
& +C_{\varepsilon_{1}}\left(1+\sigma f_{w, 2}\right) \frac{\varepsilon}{k} \tilde{P}-C_{\varepsilon_{2}} f_{\varepsilon} \frac{\varepsilon \tilde{\varepsilon}}{k} \\
& +f_{w, 2}\left[\left(\frac{7}{9} C_{\varepsilon_{2}}-2\right) \frac{\varepsilon \tilde{\varepsilon}}{k}-\frac{1}{2 k}\left(\varepsilon-\frac{2 \bar{v} k}{y^{2}}\right)^{2}\right]
\end{aligned}
$$

com

$$
\tilde{\varepsilon}=\varepsilon-2 \bar{v}\left(\frac{\partial k^{1 / 2}}{\partial x_{y}}\right)^{2}
$$

Os dois fechos da correlação das flutuações da pressão, $\phi_{i j}^{*}$, são dados pelas Eqs. (15) a (17).

$$
\phi_{i j}^{*}=\phi_{i j}=\phi_{i j, 1}+\phi_{i j, w} f_{w, 1}
$$

com

$$
\begin{aligned}
& \phi_{i j, 1}=-C_{1} \frac{\varepsilon}{k}\left(\overline{u_{i} u_{j}}-\frac{2}{3} k \delta_{i j}\right)-\alpha\left(P_{i j}-\frac{2}{3} \tilde{P} \delta_{i j}\right) \\
& -\beta\left(D_{i j}-\frac{2}{3} \tilde{P} \delta_{i j}\right)-\gamma k\left(\frac{\partial U_{i}}{\partial x_{j}}+\frac{\partial U_{j}}{\partial x_{i}}\right) \\
& \phi_{i j, w}=C_{1} \frac{\varepsilon}{k}\left(\overline{u_{i} u_{j}}-\frac{2}{3} k \delta_{i j}\right) \\
& -\frac{\varepsilon}{k}\left(\overline{u_{i} u_{k}} n_{k} n_{j}+\overline{u_{j} u_{k}} n_{k} n_{i}\right)-\alpha^{*}\left(P_{i j}-\frac{2}{3} \tilde{P} \delta_{i j}\right)
\end{aligned}
$$

Nas Eqs. (16) e (17) as várias quantidades são dadas por

$$
D_{i j}=\left[\overline{u_{i} u_{k}} \frac{\partial U_{k}}{\partial x_{j}}+\overline{u_{j} u_{k}} \frac{\partial U_{k}}{\partial x_{i}}\right],
$$

$$
\begin{aligned}
& P_{i j}=\left[\overline{u_{i} u_{k}} \frac{\partial U_{i}}{\partial x_{k}}+\overline{u_{j} u_{k}} \frac{\partial U_{i}}{\partial x_{k}}\right], \tilde{P}=\frac{1}{2} P_{i i}, \\
& \gamma=\frac{\left(30 C_{2}-2\right)}{55}, \beta=\frac{\left(8 C_{2}-2\right)}{11}, \alpha=\frac{\left(8+C_{2}\right)}{11},
\end{aligned}
$$

O efeito dos números de Reynolds no modelo da distribuição de pressão é realizado através da função de $f_{w, 1}$, definida na Eq. (20), que incorpora os efeitos reológicos, nomeadamente o efeito reofluidificante da viscosidade de corte e o reoespessante da razão de Trouton.

$$
\begin{aligned}
& f_{w, 1}=\exp \left[-\left[2.5\left\{1-\left[1+\left|\frac{1-n}{1+n}\right| y^{+}\right]^{-1+n / 1-n \mid / A^{+}}\right\}\right.\right. \\
& \left.\times\left\{1-\left[1+\left|\frac{p-1}{3-p}\right| y^{+} C^{\frac{1-p}{2-p}}\right]^{-3-p / p-1 / A^{+}} \mid\right)^{1.1}\right]
\end{aligned}
$$

Os restantes parâmetros e funções de amortecimento do modelo das tensões de Reynolds são apresentados na Tabela 1 .

Tabela 1. Parâmetros e funções de amortecimento do modelo das tensões de Reynolds

\begin{tabular}{|c|c|c|}
\hline \multicolumn{3}{|c|}{ Parâmetros } \\
\hline$C_{1}=1.5$ & $C_{2}=0.4$ & $\alpha^{*}=0.45$ \\
\hline$C_{\varepsilon_{1}}=1.35$ & $C_{\varepsilon_{2}}=1.8$ & $C_{s}=0.11$ \\
\hline$C=25$ & $C_{V 1}=-1.7$ & $C_{V 2}=0.2$ \\
\hline \multicolumn{2}{|c|}{ Funções de amortecimento } \\
\hline \multicolumn{3}{|c|}{$f_{w, 2}=\exp \left[-\left(R_{T} / 64\right)^{2}\right]$} \\
\hline \multicolumn{2}{|c|}{$f_{\varepsilon}=1-(2 / 9) \exp \left[-\left(R_{T} / 6\right)^{2}\right]$} \\
\hline
\end{tabular}

O número turbulento de Reynolds $R_{T}$ é definido por $R_{T}=k^{2} /(\overline{v \varepsilon})$.

\section{Comparação com fluidos FENE-P}

O programa usado para realizar as simulações numéricas de escoamentos turbulentos desenvolvidos em conduta é baseado na discretização de volumes finitos das equações governativas e das equações do modelo turbulento. Para o acoplamento entre a pressão e a velocidade é utilizado o método SIMPLEC, e na resolução da matriz das equações discretizadas é usado o algoritmo TDMA para sistemas unidimensionais. A malha usada é não uniforme com 199 células ao longo da seção da conduta, com previsões independentes da malha para fluidos newtonianos e não newtonianos, dentro de uma margem de $0.1 \%$. O domínio de simulação é exclusivamente na direção transversal com as seguintes imposições de fronteira na parede: 


$$
U_{i}=0 ; \overline{u_{i} u_{j}}=0 \text { e } \varepsilon=2 \bar{v}\left(\frac{\partial k^{1 / 2}}{\partial y}\right)^{2} \text { para } r=R
$$

Como já referido na introdução, nos novos desenvolvimentos na modelação de turbulência para fluidos viscoelásticos, a reologia dos fluidos foi representada pelo o modelo FENE-P, usando os dados do DNS. Contudo, as previsões de DNS de diferentes modelos de FENE-P representando a reologia dos fluidos não correspondem quantitativamente aos resultados experimentais, descrito por Ptasinski et al. (2003).

Nesse sentido, para analisar o desempenho de um modelo GNF contra o modelo FENE-P, comparamos as previsões do modelo turbulento de tensões de Reynolds para fluidos viscoelásticos, de Resende et al. (2013a), com a parte experimental e simulação DNS apresentada por Ptasinski et al. (2003). Numa primeira fase foi necessário quantificar os parâmetros reológicos, e para isso ajustou-se o modelo GNF modificado à reologia do fluido representada pelo modelo FENE-P de Ptasinski et al. (2003), designada por Run A e com os seguintes parâmetros: $L=10$, $\beta=0.6, W e_{\tau_{0}}=54, R e_{w}=8609$ e $\eta_{w} / \eta_{0}=0.792$.

A viscosidade de corte e extensional do modelo FENE-P é dado por Purnode e Crochet (1998). Na Figura 1 (a) - (b) é apresentando a viscosidade de corte e a razão de Trouton, respectivamente, para ambos os modelos GNF e FENE-P. O modelo GNF é ajustado à viscosidade de corte para determinar os parâmetros $n$ e $K_{v}$, apenas na região dos dados medidos, como é mostrado na Figura 1 (a). No caso da viscosidade extensional, esta é relacionada à razão de Trouton através da relação $\operatorname{Tr}=\eta_{e}(\dot{\varepsilon}) / 3 \eta_{v}(\dot{\gamma})$. Como pode ser observado na Figura 1 (b), o modelo FENE-P apresenta dois patamares com uma variação abrupta, enquanto o modelo GNF não consegue prever esse efeito. Por essa razão, é efetuado um ajuste de lei de potência para o intervalo de $10^{-4}$ a $10^{+5} \mathrm{~s}^{-1}$ da taxa de deformação, obtendo assim os parâmetros $p$ e $K_{e}$ (mais detalhes podem ser encontrados em Cruz e Pinho (2003))

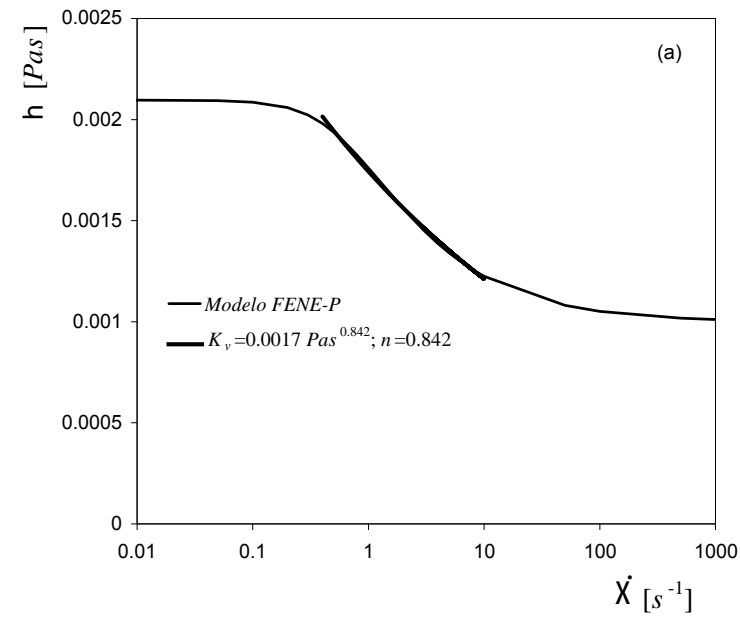

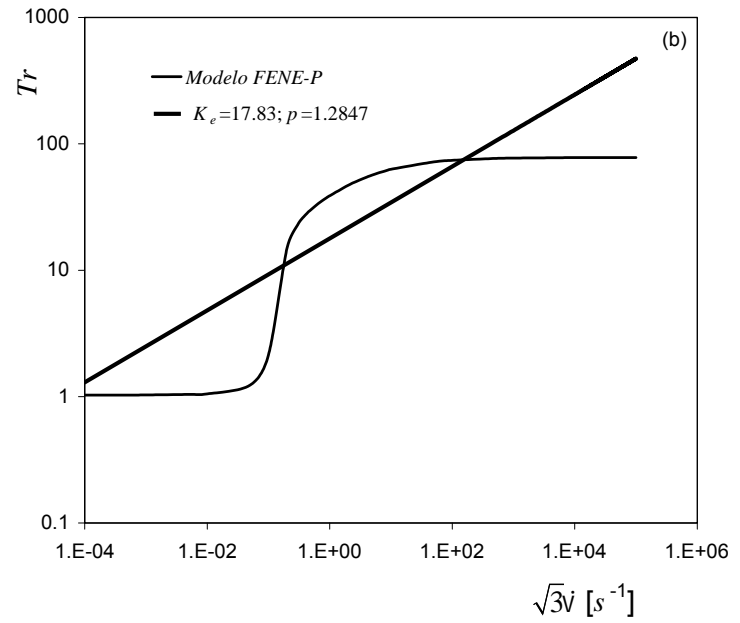

Figura 1. Comparação entre as viscosidades representadas pelos modelos GNF modificado e FENE-P: (a) viscosidade de corte; (b) razão de Trouton.

Figura 2 compara as previsões do perfil de velocidade, de um escoamento turbulento entre placas, com o modelo de tensões de Reynolds stress usando o ajuste do modelo GNF, mostrado nas Figura 1 (a) - (b), com os dados experimntais e as simulações de DNS de Ptasinski et al. (2003). O modelo de tensões de Reynolds prevê satisfatoriamente os dados experimentais. Apesar das previsões corresponderem bem aos dados apresentados do perfil de velocidade existe uma sobreprevisão do valor da redução de arrasto, $\mathrm{DR}=32 \%$, em comparação com os dados experimentais, $\mathrm{DR}=23 \%$. Da mesma forma, comparando os dados experimentais de Ptasinski et al. (2003) com os dados de DNS de Li et al. (2006b), equivalente a $\mathrm{DR}=29 \%$, verificamos que os perfis de velocidade coincidem, mas com diferentes DR, $\mathrm{DR}=29 \%$ contra $\mathrm{DR}=23 \%$ de Ptasinski et al. (2003).

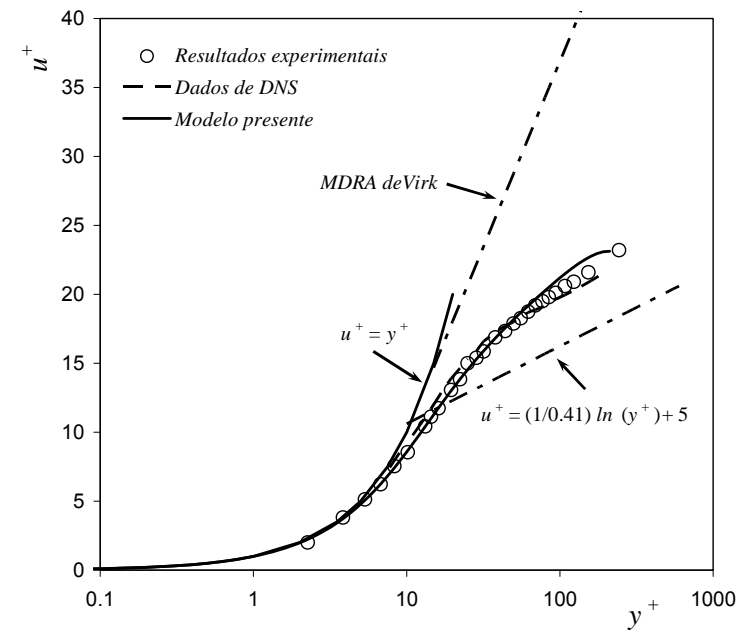

Figura 2. Comparação entre as previsões do modelo de tensões de Reynolds (linha contínua), as previsões de DNS (linha tracejada) e os dados experimentais (símbolos) de Ptasinski et al. (2003). 


\section{Conclusões}

Foi analisado o desempenho do modelo de turbulência de tensões de Reynolds de Resende et al. (2013a), as previsões de um escoamento turbulento entre placas foram comparadas com os dados experimentais, e as previsões de simulação numérica direta (DNS) baseadas na equação constitutiva reológica FENE-P. As previsões do DNS conseguem prever bem as medições de redução do arrasto, porque os parâmetros do modelo FENE-P, em particularmente o fator de relaxação, foram ajustados com esse objetivo, de obter o mesmo DR. Contudo é de referir que não foi ajustado às medições experimentais do tempo de relaxação. No caso do modelo GNF, em que o ajuste foi efetuado diretamente à viscosidade de corte e à razão de Trouton do fluido, foi possível obter boas previsões, apesar de serem inferiores às do DNS.

O modelo de tensões de Reynolds de Resende et al. (2013a) representa um avanço significativo quando comparamos com os modelos anteriores de duas equações, todavia para obter uma análise total do seu desempenho é necessário no futuro estender a diferentes regimes da redução de arrasto e a geometrias complexas. E com base nas previsões do modelo, demonstradas no presente artigo, acredita-se que o seu desempenho será melhor que os modelos anteriores de Cruz et al. (2004); Resende et al. (2006).

\section{Agradecimentos}

$\mathrm{O}$ autor P. R. Resende quer agradecer ao financiamento da FAPESP através do projeto 2013/01521-4.

\section{Referências Bibliográficas}

Barnes, H.A., Hutton, J.F., Walters, K., 1989. An Introduction to Rheology, Amsterdam.

Cruz, D.O.A., Pinho, F.T., 2003. Turbulent pipe flow predictions with a low Reynolds number $k$ - $\varepsilon$ model for drag reducing fluids. Journal of NonNewtonian Fluid Mechanics, Vol. 114, pp. 109148. DOI: 10.1016/S0377-0257(03)00119-8

Cruz, D.O.A., Pinho, F.T., Resende, P.R., 2004. Modeling the new stress for improved drag reduction predictions of viscoelastic pipe flow. Journal of Non-Newtonian Fluid Mechanics, Vol. 121, pp. 127-141. DOI:

10.1016/j.jnnfm.2004.05.004

Dimitropoulos, C.D., Sureshkumar, R., Beris, A.N., Handler, R.A., 2001. Budgets of Reynolds stress, kinetic energy and streamwise entrophy in viscoelastic turbulent channel flow. Physics of Fluids, Vol. 13, pp. 1016-1027. DOI: $10.1063 / 1.1345882$

Dubief, Y., Laccarino, G., Lele, S.K., 2004. A turbulence model for polymer flows. Center for Turbulence Research, Stanford.
Durst, F., Rastogi, A.K., 1977. Calculations of turbulent boundary layer flows with drag reducing polymer additives. Physics of Fluids, Vol. 20, pp. 1975-1985. DOI: 10.1063/1.861827

Hassid, S., Poreh, M., 1978. A turbulent energy dissipation model for flows with drag reduction. Journal of Fluids Engineering, Vol. 100, pp. 107-112. DOI: $10.1115 / 1.3448580$

Iaccarino, G., Shaqfeh, E.S.G., Dubief, Y., 2010. Reynolds-averaged modeling of polymer drag reduction in turbulent flows. Journal of NonNewtonian Fluid Mechanics, Vol. 165, pp. 376384. DOI: $10.1016 /$ j.jnnfm.2010.01.013

Li, C.F., Gupta, V.K., Sureshkmar, R., Khomami, B., 2006a. Turbulent channel flow of dilute polymeric solutions: drag reduction scaling and an eddy viscosity model. Journal of NonNewtonian Fluid Mechanics, Vol. 139, pp. 177189. DOI: $10.1016 /$ j.jnnfm.2006.04.012

Li, C.F., Sureshkmar, R., Khomami, B., 2006 b. Influence of rheological parameters on polymer induced turbulent drag reduction. Journal of Non-Newtonian Fluid Mechanics, Vol. 140, pp. 23-40. DOI: 10.1016/j.jnnfm.2005.12.012

Mizushina, T., Usui, H., Yoshida, T., 1973. Turbulent pipe flow of dilute polymer solutions. Journal Chem. Eng. Japan, Vol. 7, pp. 162-167. DOI: 10.1252 jcej.7.162

Pinho, F.T., 2003. A GNF framework for turbulent flow models of drag reducing fluids and proposal for a $k$ - $\varepsilon$ type closure. Journal of Non-Newtonian Fluid Mechanics, Vol. 114, pp. 149-184. DOI: 10.1016/S0377-0257(03)00120-4

Pinho, F.T., Li, C.F., Younis, B.A., Sureshkumar, R., 2008. A low Reynolds number k- eturbulence model for FENE-P viscoelastic fluids. Journal of Non-Newtonian Fluid Mechanics, Vol. 154, pp. 89-108. DOI: 10.1016/j.jnnfm.2008.02.008

Poreh, M., Hassid, S., 1977. Mean velocity and turbulent energy closures for flows with drag reduction. Physics of Fluids, Vol. 20, pp. 193196. DOI: $10.1063 / 1.861729$

Ptasinski, P.K., Boersma, B.J., Nieuwstadt, F.T.M., Hulsen, M.A., Brule, B.H.A.A.V.D., Hunt, J.C.R., 2003. Turbulent channel flow near maximum drag reduction: simulation, experiments and mechanisms. Journal of Fluid Mechanics, Vol. 490, pp. 251-291. DOI: $10.1017 /$ S0022112003005305

Purnode, B., Crochet, M.J., 1998. Polymer solution characterization with the FENE-P model. Journal of Non-Newtonian Fluid Mechanics, Vol. 77, pp. 1-20. DOI: 10.1016/S0377-0257(97)00096-7

Resende, P.R., Escudier, M.P., Presti, F., Pinho, F.T., Cruz, D.O.A., 2006. Numerical predictions and measurements of Reynolds normal stresses in turbulent pipe flow of polymers. Int. Journal of Heat and Fluid Flow, Vol. 27, pp. 204-219. DOI: 10.1016/j.ijheatfluidflow.2005.08.002 
Resende, P.R., Kim, K., Younis, B.A., Sureshkumar, R., Pinho, F.T., 2011. A $k-\varepsilon$ turbulence model for FENE-P fluid flows at low and intermediate regimes of polymer-induced drag reduction. Journal of Non-Newtonian Fluid Mechanics, Vol. 166, pp. 639- 660. DOI:

10.1016/j.jnnfm.2011.02.012

Resende, P.R., Pinho, F.T., Cruz, D.O.A., 2013a. A Reynolds stress model for turbulent flows of viscoelastic fluids. Submitted to Journal of Turbulence.

Resende, P.R., Pinho, F.T., Younis, B.A., Kim, K., Sureshkumar, R., 2013b. Development of a lowReynolds-number k- $\omega$ model for FENE-P fluids. Flow, Turbulence and Combustion, Vol. 90, pp. 69-94. DOI: 10.1007/s10494-012-9424-x

Sureshkumar, R., Beris, A.N., Handler, R.A., 1997. Direct numerical simulation of the turbulent channel flow of a polymer solution. Physics of Fluids, Vol. 9, pp. 743-755. DOI: $10.1063 / 1.869229$ 